\title{
Multimodality approach to mediastinal staging in non-small cell lung cancer. Faults and benefits of PET-CT: a randomised trial
}

\author{
Barbara M Fischer, ${ }^{1,2}$ Jann Mortensen, ${ }^{1}$ Hanne Hansen, ${ }^{3}$ Peter Vilmann, ${ }^{4}$ \\ Søren S Larsen, ${ }^{5}$ Annika Loft, ${ }^{1}$ Anne K Bertelsen, ${ }^{1}$ Jesper Ravn, ${ }^{6}$ Paul Clementsen, \\ Asbjørn Høegholm, ${ }^{8}$ Klaus R Larsen, ${ }^{9}$ Asger Dirksen, ${ }^{7}$ Birgit G Skov, ${ }^{10}$ Mark Krasnik, ${ }^{11}$ \\ Liselotte Højgaard, ${ }^{1}$ Ulrik Lassen ${ }^{12}$
}

\section{See Editorial, p 275}

1 Department of Clinical Physiology, Nuclear Medicine and PET, Rigshospitalet, Copenhagen University Hospital, Copenhagen, Denmark 2Department of Clinical Physiology and Nuclear Medicine, Hvidovre Hospital, Hvidovre, Denmark ${ }^{3}$ Department of Radiology, Bispebjerg Hospital,

Copenhagen, Denmark ${ }^{4}$ Department of Surgery, Herlev Hospital, Endoscopic Unit at Gentofte Hospital, Hellerup, Denmark

${ }^{5}$ Department of Surgery, Hvidovre Hospital, Hvidovre, Denmark

${ }^{6}$ Department of Thoracic Surgery, Rigshospitalet, Copenhagen University Hospital Copenhagen, Denmark ${ }^{7}$ Department of Pulmonology, Gentofte Hospital, Hellerup, Denmark

${ }^{8}$ Department of Pulmonology, Naestved Hospital, Næstved, Denmark

${ }^{9}$ Department of Pulmonology, Bispebjerg Hospital,

Copenhagen, Denmark

${ }^{10}$ Department of Pathology,

Bispebjerg Hospital, Copenhagen, Denmark

${ }^{11}$ Department of Development and Quality, Copenhagen University Hospital,

Copenhagen, Denmark

${ }^{12}$ Department of Oncology, Rigshospitalet, Copenhagen University Hospital, Copenhagen, Denmark

\section{Correspondence to} Barbara M Fischer, Department of Clinical Physiology and Nuclear Medicine, Hvidovre Hospital, section 239, Kettegaard Allé 30, 2650 Hvidovre, Denmark: bjerregaard.fischer@gmail.com

Received 4 November 2010 Accepted 11 November 2010 Published Online First 17 December 2010

\begin{abstract}
Background Correct mediastinal staging is a cornerstone in the treatment of patients with non-small cell lung cancer. A large range of methods is available for this purpose, making the process of adequate staging complex. The objective of this study was to describe faults and benefits of positron emission tomography (PET)-CT in multimodality mediastinal staging.
\end{abstract}

Methods A randomised clinical trial was conducted including patients with a verified diagnosis of non-small cell lung cancer, who were considered operable. Patients were assigned to staging with PET-CT (PET-CT group) followed by invasive staging (mediastinoscopy and/or endoscopic ultrasound with fine needle aspiration (EUS-FNA)) or invasive staging without prior PET-CT (conventional work up (CWU) group). Mediastinal involvement (dichotomising $\mathrm{N}$ stage into $\mathrm{NO}-1$ versus N2-3) was described according to CT, PET-CT, mediastinoscopy, EUS-FNA and consensus (based on all available information), and compared with the final $\mathrm{N}$ stage as verified by thoracotomy or a conclusive invasive diagnostic procedure.

Results A total of 189 patients were recruited, 98 in the PET-CT group and 91 in the CWU group. In an intentionto-treat analysis the overall accuracy of the consensus $\mathrm{N}$ stage was not significantly higher in the PET-CT group than in the CWU group $190 \%$ (95\% confidence interval $82 \%$ to $95 \%$ ) vs $85 \%(95 \% \mathrm{Cl} 77 \%$ to $91 \%))$. Excluding the patients in whom PET-CT was not performed $(n=14)$ the difference was significant $195 \%(95 \% \mathrm{Cl} 88 \%$ to $98 \%$ ) vs $85 \%$ ( $95 \% \mathrm{Cl} 77 \%$ to $91 \%$ ), $p=0.034$ ). This was mainly based on a higher sensitivity of the staging approach including PET-CT.

Conclusion An approach to lung cancer staging with PET-CT improves discrimination between N0-1 and N2-3. In those without enlarged lymph nodes and a PET-negative mediastinum the patient may proceed directly to surgery. However, enlarged lymph nodes on CT needs confirmation independent of PET findings and a positive finding on PET-CT needs confirmation before a decision on surgery is made.

Clinical trial number NCT00867412.

\section{INTRODUCTION}

Staging is a complex and critical event in the care of patients with lung cancer; precise description of the extent of the disease is important for the selection of the proper treatment modality as well as in predicting prognosis. ${ }^{1}$

In patients without distant metastases, the most significant factor for deciding treatment is the status of mediastinal lymph nodes, as mediastinal spread (N2-3 disease) excludes the patient from primary surgery. The European Society of Thoracic Surgery (ESTS) as well as the American College of Chest Physicians (ACCP) has published guidelines for proper preoperative mediastinal staging, ${ }^{2-4}$ and there seems to be a cross-continent consensus on the approach to preoperative staging. However, scratching the surface of the consensus reveals several unresolved problems and controversies:

1. Positron emission tomography (PET)-CT is recommended if available. ${ }^{2} 3$ However, two randomised clinical trials have demonstrated that staging by means of PET-CT significantly reduces the number of thoracotomies as well as the frequency of futile thoracotomies. ${ }^{5}$ Is this only due to detection of unknown distant metastases or does PET-CT also increase the diagnostic accuracy of mediastinal staging?

2. It has been suggested that mediastinoscopy or other invasive staging can be omitted in certain cases where mediastinum is PET negative. ${ }^{2}{ }^{4}$ However, recent data suggest that by doing this, $16 \%$ of these patients have occult N2 disease. ${ }^{7}$

3. Does the combination of knowledge of lymph node size on CT and $\left[{ }^{18} \mathrm{~F}\right]$ fluorodeoxyglucose (FDG) accumulation on PET increase the diagnostic accuracy of PET ? ${ }^{8}$

4. Does mediastinoscopy increase diagnostic accuracy in the case of positive imaging and negative endoscopic ultrasound (EUS) $?^{2}$

The purpose of the current study is to suggest answers to and discuss these problems, based on results from a randomised clinical trial. ${ }^{5}$

\section{METHODS}

\section{Setting and participants}

Patients were recruited from three departments of pulmonology in the area of Copenhagen, Denmark. Patients were between 18 and 80 years of age with a newly diagnosed non-small cell lung cancer (NSCLC) and considered operable after conventional staging procedures. ${ }^{5} 9$ The study was approved by the Ethics Committee and Institutional Review Board of Copenhagen hospitals 
(reference 11-118/01 and 01-252/00) and conducted according to the Declarations of Helsinki and Tokyo (http://ClinicalTrials.gov number, NCT00867412). Written informed consent was obtained from all patients.

\section{Randomisation and intervention}

After initial CT, eligible patients were 1:1 randomly assigned to (1) PET-CT followed by invasive diagnostic procedures (PET-CT group) or (2) invasive diagnostic procedures alone (conventional work-up (CWU) group). Randomisation was done centrally by a permuted block design, stratified by sex and recruiting centre. Standard staging procedures were governed by local routine based on current guidelines; however, mediastinoscopy was considered mandatory. TNM stage was assigned according to the revised Mountain classification. ${ }^{10}$

\section{PET-CT imaging}

All PET-CT scans were performed in the Department of Clinical Physiology, Nuclear Medicine and PET, Rigshospitalet, Copenhagen University Hospital. After a fasting period of $6 \mathrm{~h}$, $400 \mathrm{MBq}$ of FDG was given intravenously and the patient rested for $1 \mathrm{~h}$. The patient was scanned from the head to the upper thigh on an integrated PET-CT system (GE Discovery LS, General Electric Medical Systems, Milwaukee, Wisconsin, USA). A diagnostic CT protocol with intravenous contrast was applied. ${ }^{5}$ PET-CT images were evaluated side by side by an experienced radiologist and nuclear medicine specialist.

\section{Invasive staging}

Mediastinoscopy was performed in all patients regardless of CT and PET-CT findings, unless this procedure was contraindicated for other reasons. Specialists in cardiothoracic surgery or laryngologists performed the mediastinoscopy under general anaesthesia. Material from regions $2 / 4 \mathrm{R}, 2 / 4 \mathrm{~L}$ and 7 were sampled if visible or palpable. ${ }^{10}$ At one trial site a randomised trial evaluating the value of EUS with fine needle aspiration (EUS-FNA) was ongoing, ${ }^{11}$ thus approximately half of the patients had an EUS-FNA procedure performed, either during the same general anaesthesia as used for mediastinoscopy (EUS-FNA first) or as an outpatient procedure with the patient under conscious sedation. The EUS examination was performed with an electronic linear transducer with an adjustable ultrasonic frequency of 5 or $7.5 \mathrm{MHz}$ and with a penetration depth of $7-8 \mathrm{~cm}$ (Olympus ultrasonic endoscope (GF-UC160P-OL5) or Pentax EG 3870). All lymph nodes with at least one criterion suggestive of malignancy were sampled (22 gauge needle, MEDIGlobe, type Sono-tip 2). The cytological specimens were stained by the May-Grünwald-Giemsa method and examined by an experienced pathologist. Only results from EUS-FNA procedures were used for staging and reported in the following. At the same site, prototype equipment for endobronchial ultrasoundguided transbronchial needle aspiration (EBUS-TBNA) was under development, and some patients also underwent this procedure.

At thoracotomy, samples were taken from all available mediastinal and hilar lymph node stations. $\mathrm{N}$ stage defined by the pathologist after thoracotomy served as the reference $(\mathrm{N}$ final). For patients in whom thoracotomy was not performed, $\mathrm{N}$ stage assigned by mediastinoscopy, EUS-FNA or EBUS-TBNA served as the reference. If imaging modalities suggested $\mathrm{N} 2-3$ disease and this could not be confirmed by mediastinoscopy or any other modality and the patient did not have a thoracotomy, an $\mathrm{N}$-final stage was not assigned (Nx).

\section{Outcomes and follow-up}

Before the decision was made to operate, a consensus TNM stage was assigned by a pulmonologist and a thoracic surgeon on the basis of all available information (clinical data, initial CT, PETCT, bronchoscopy, mediastinoscopy and, if available, EUS-FNA or EBUS-TBNA). Mediastinoscopy, EUS-FNA or EBUS-TBNA served as the gold standard when assessing mediastinal lymph node involvement (consensus $\mathrm{N}$ stage). Positive findings on PETCTwere evaluated by histology or other imaging modality at the discretion of the referring clinician. Follow-up data were retrieved from patient files and the local patient registry.

\section{Statistical analysis}

The primary analysis on diagnostic accuracy of a staging approach with and without PET-CT was done as an intentionto-treat analysis, including all randomised patients with an $\mathrm{N}$ final stage. For the purpose of comparing the performance of individual modalities, only data on patients in whom the relevant examination was performed were included. Diagnostic accuracy, sensitivity, specificity, positive predictive value (PPV) and negative predictive value (NPV) with corresponding 95\% CIs (Wilson score method without continuity correction) were calculated. The variables were compared by hypothesis testing for proportions in two independent groups, assuming normal distribution of the differences and without continuity correction. ${ }^{12}$ Clinical characteristics were compared with the use of an independent $\mathrm{t}$ test for continuous variables (age) and a $\chi^{2}$ test or Fisher exact test for mutually exclusive categorical variables (sex, histology, $\mathrm{N}$-final and confirmation of $\mathrm{N}$-final). Proportions of patients in the two groups on whom different staging procedures were performed were compared by hypothesis testing as described above. Agreement between a modality and $\mathrm{N}$-final within a patient group (correlated data) was measured by Cohens kappa ( $\kappa)$ and McNemars test. Kappa values were interpreted as suggested by Altman: $<0.20$ poor agreement; $0.21-0.40$ fair agreement; $0.41-0.60$ moderate agreement; $0.61-0.80$ good agreement; and 0.81-1.00 excellent agreement. ${ }^{13}$ All reported $p$ values are two-sided and a significance level of 0.05 was applied. Statistical analysis was performed with the use of SPSS software, version 16.

\section{RESULTS}

\section{Patients}

A total of 189 patients were recruited to the trial (98 in the PETCT group and 91 in the CWU group, figure 1). CT was performed in all patients. A PET-CT scan was not performed in 14 patients in the PET-CT group due to an unacceptably long waiting time or technical problems with the PET-CT equipment. Patient characteristics were well balanced (table 1). Mediastinoscopy was performed in 89 patients $(91 \%)$ in the PET-CT group and 88 (97\%) in the CWU group. On average 1.3 and 1.4 biopsies from N2 lymph nodes and 0.5 and 0.6 biopsies from N3 lymph nodes were performed in each patient in the PET-CT and CWU group, respectively. EUS-FNA was performed in 40 (41\%) and $29(32 \%)$ patients, respectively, in each group. In the PETCT group, an FNA from lymph node station 7 was performed in $70 \%$ of the procedures and an FNA from at least one of the lymph nodes stations $1,2-4 \mathrm{R} / \mathrm{L}, 5-6$ or 8-9 was performed in $39 \%$ of the procedures. The values in the CWU group were $90 \%$ and $21 \%$, respectively (table 2 ).

\section{Diagnostic accuracy}

Diagnostic accuracy of PET-CT, CT, mediastinoscopy and EUSFNA in assigning correct $\mathrm{N}$ stage ( $\mathrm{N} 0-1$ vs $\mathrm{N} 2-3$ ) is described in 
Figure 1 Study flow diagram.

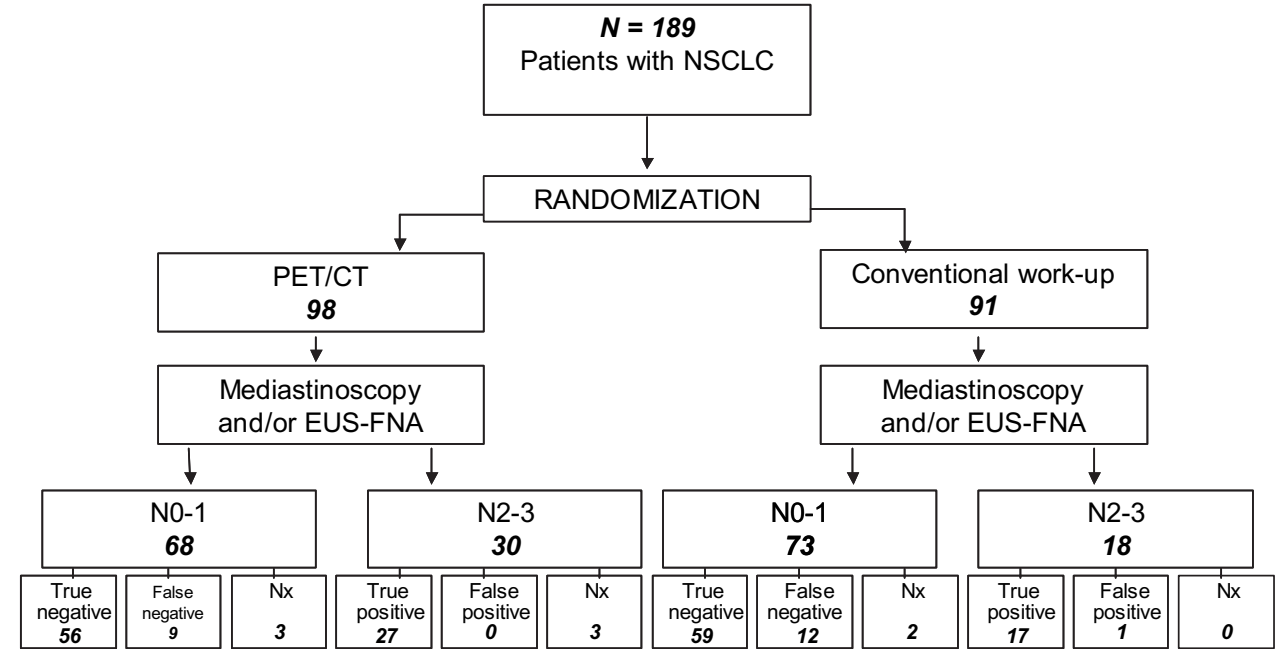

$\mathrm{Nx}$ : A final N-stage could not be assigned
Appendix 1. Consensus $\mathrm{N}$ stage is the $\mathrm{N}$ stage assigned based on all available information prior to decision on surgery. $\mathrm{N}$-final served as the reference, excluding six and two patients, respectively, in each group due to lack of confirmation of the final $\mathrm{N}$ stage $(\mathrm{Nx})$. By intention-to-treat analysis (table 3 ) the accuracy of the staging strategy with PET-CT appears only slightly superior to the CWU staging strategy $(90 \%$ (95\% CI $82 \%$ to $95 \%$ ) vs $85 \%$ (95\% CI $77 \%$ to $91 \%), p=0.322)$, mainly based on

Table 1 Patient characteristics

\begin{tabular}{|c|c|c|c|}
\hline Patient & РET-CT $(n=98)$ & CWU $(n=91)$ & p Value \\
\hline Mean age in years (range) & $62(42-80)$ & $64(38-80)$ & 0.222 \\
\hline Sex (male (\%)) & $53(54)$ & $49(54)$ & 0.974 \\
\hline \multicolumn{4}{|l|}{ Staging procedures, $\mathrm{n}(\%)$} \\
\hline СT & $98(100)$ & $91(100)$ & - \\
\hline PET-CT & $84(86)$ & $0(0)$ & $<0.001$ \\
\hline Mediastinoscopy & $89(91)$ & $88(97)$ & 0.097 \\
\hline EUS & $47(48)$ & $35(38)$ & 0.187 \\
\hline EUS-FNA & $40(41)$ & $29(32)$ & 0.201 \\
\hline EBUS & $8(8)$ & $11(12)$ & 0.368 \\
\hline \multicolumn{4}{|c|}{ Histology at operation $(n=60 / n=73)$} \\
\hline Adenocarcinoma & 29 & 29 & 0.330 \\
\hline Squamous cell carcinoma & 20 & 22 & \\
\hline Large cell carcinoma & 4 & 12 & \\
\hline Bronchioalveolar carcinoma & 0 & 1 & \\
\hline NSCLC, other & 7 & 6 & \\
\hline Benign & 0 & 3 & \\
\hline \multicolumn{4}{|l|}{ Final $\mathrm{N}$ stage } \\
\hline No-1 & 56 & 60 & 0.268 \\
\hline $\mathrm{N} 2-3$ & 36 & 29 & \\
\hline $\mathrm{Nx}$ & $6^{*}$ & $2 \dagger$ & \\
\hline \multicolumn{4}{|l|}{ Final N stage confirmed by $(n)$} \\
\hline Thoracotomy & 60 & 72 & 0.014 \\
\hline Mediastinoscopy & 9 & 10 & \\
\hline EUS-FNA & 19 & 7 & \\
\hline EBUS-TBNA & 4 & 0 & \\
\hline Not applicable & $6^{*}$ & $2 \dagger$ & \\
\hline
\end{tabular}

*In 4 patients M1 was found on PET-CT and further confirmation of $\mathrm{N}$ stage was not sought. One patient was No-1, but considered inoperable due to co-existing disease. Explorative thoracotomy was performed in one patient due to inoperable T4 disease; $\mathrm{N}$ stage not confirmed.

†Explorative thoracotomy was performed in two patients due to inoperable T4 disease; $\mathrm{N}$ stage not confirmed.

CWU, conventional work-up; EBUS, endobronchial ultrasound; EUS, endoscopic ultrasound; FNA, fine needle aspiration; NSCLC, non-small cell lung cancer; PET, positron emission tomography; TBNA, transbronchial needle aspiration. an improved sensitivity $(75 \%$ (95\% CI $59 \%$ to $86 \%)$ vs $59 \%(95 \%$ CI $41 \%$ to $74 \%$ ), $\mathrm{p}=0.162$ ). Excluding the 14 patients in the PETCT group on whom a PET-CT scan was not performed, the diagnostic accuracy of the consensus $\mathrm{N}$ stage was significantly higher in the PET-CT group compared with the CWU group (difference of $10 \%$ (95\% CI $0.2 \%$ to $20 \%$ ), $p=0.034$, table 3 ), again primarily based on the improved sensitivity as both groups had equally high specificity, based on the results of the invasive staging methods.

In 82 patients in the PET-CT group an exact description of the localisation (central, intermediate and peripheral) of the primary tumour on initial CT scan was available (table 4). In patients with a central tumour $(n=24)$ the agreement between $N$ stage on PET-CT and N-final was fair $(\kappa=0.39)$, whereas it was moderate in patients with a peripheral or intermediate tumour (kappa $=0.54$ ). There was no systematic disagreement between $\mathrm{N}$ at PET-CT and $\mathrm{N}$-final (eg, consequent overstaging or understaging) in either subgroup (McNemar, $p=1.0$ ).

\section{PET-negative lymph nodes}

In the PET-CT group 53 (54\%) patients were staged N0-1 on PET-CT (table 5). Mediastinoscopy was performed in 49 of these patients, one with a positive finding (N2 disease). Of the remaining 48 patients who were PET negative and negative on mediastinoscopy, six patients had mediastinal metastases (three confirmed by EUS-FNA, two by thoracotomy and one by EBUSTBNA). Twenty patients with N0-1 on PET-CT had EUS-FNA

Table 2 Details on invasive procedures

\begin{tabular}{lllll}
\hline No. of nodes (mean) & PET-CT & Range & CWU & Range \\
\hline Mediastinoscopy & $\mathrm{N}=89$ & & $\mathrm{~N}=88$ & \\
N2 nodes evaluated & 1.3 & $(0-7)$ & 1.4 & $(0-7)$ \\
N3 nodes evaluated & 0.5 & $(0-3)$ & 0.6 & $(0-4)$ \\
Thoracotomy & $\mathrm{N}=60$ & & $\mathrm{~N}=73$ & \\
N1 nodes evaluated & 4.1 & $(0-19)$ & 3.8 & $(0-13)$ \\
N2 nodes evaluated & 3.4 & $(0-17)$ & 3.6 & $(0-16)$ \\
EUS-FNA & $\mathrm{N}=40$ & & $\mathrm{~N}=29$ & \\
1 & 0.03 & $(0-1)$ & 0 & - \\
2-4R/L & 0.3 & $(0-3)$ & 0.1 & $(0-2)$ \\
$5-6$ & 0 & - & 0.03 & $(0-1)$ \\
7 & 1.0 & $(0-3)$ & 1.2 & $(0-2)$ \\
$8-9$ & 0.1 & $(0-2)$ & 0.2 & $(0-3)$ \\
\hline
\end{tabular}

CWU, conventional work-up; EUS, endoscopic ultrasound; FNA, fine needle aspiration; PET, positron emission tomography. 
Table $32 \times 2$ tables and diagnostic accuracy for consensus $N$ stage

\begin{tabular}{|c|c|c|c|c|c|c|c|c|c|}
\hline PET-CT group I* & \multicolumn{3}{|c|}{ N-Final } & \multicolumn{2}{|c|}{ CWU group* } & \multicolumn{3}{|c|}{ N-Final } & \\
\hline Consensus N-stage & N0-1 & $\mathrm{N} 2-3$ & Total & Consens & us N-sta & ge $\mathrm{N} 0-1$ & N2-3 & 3 Total & \\
\hline No-1 & 56 & 9 & 65 & & NO & 59 & 12 & 71 & \\
\hline N2-3 & 0 & 27 & 27 & & N2 & -3 & 17 & 18 & \\
\hline Total & 56 & 36 & 92 & & Tot & 60 & 29 & 89 & \\
\hline DCT OT n & & & & & CWU & PET-CT I & $\mathrm{p}$ & PET-CT II & $\mathrm{p}$ \\
\hline PET-CT group $\|^{\prime}$ & N-Fin & & & Sensitivity & $59 \%$ & $75 \%$ & 0.162 & $85 \%$ & 0.034 \\
\hline Consensus $\mathrm{N}$-stage & No-1 & $\mathrm{N} 2-3$ & Total & & $(41-74)$ & $(59-86)$ & & $(66-94)$ & \\
\hline No-1 & 53 & 4 & 57 & Specificity & $98 \%$ & $100 \%$ & 0.337 & $100 \%$ & 0.347 \\
\hline N2-3 & 0 & 22 & 22 & & $(91-100)$ & $(94-100)$ & & $(93-100)$ & \\
\hline Total & 53 & 26 & 79 & Accuracy & $\begin{array}{l}85 \% \\
\end{array}$ & $\begin{array}{l}90 \% \\
\end{array}$ & 0.322 & $\begin{array}{l}95 \% \\
\end{array}$ & 0.040 \\
\hline
\end{tabular}

*Intention-to-treat analysis, all randomised patients with final N-stage.

${ }^{\dagger}$ Only patients who performed PET-CT and to whom a final N-stage could be assigned.

performed. The cytology showed N2-3 disease in four of these patients and was false negative in one patient. In patients with N0-1 on PET-CT, agreement between mediastinoscopy and Nfinal was fair $(\kappa=0.22)$ with a tendency to underdiagnose (McNemar, $\mathrm{p}=0.031$ ). Agreement between EUS-FNA and $\mathrm{N}$ final was excellent $(\kappa=0.857)$. Overall PET-CT gave a falsenegative result in eight patients (false-negative rate, 8/53 15\% $(95 \%$ CI $8 \%$ to $27 \%))$. Two of the latter patients proceeded to thoracotomy, and inspection of the surgical specimen (macroscopic as well as microscopic) revealed carcinoma in lymph node station 7 , and in 7 and $2 \mathrm{~L}$, respectively.

\section{Lymph node size}

In table 6 the results of PET-CT are categorised according to the size of mediastinal lymph nodes on CT $(<10 \mathrm{~mm}$ or $\geq 10 \mathrm{~mm})$. The diagnostic accuracy of PET-CT was $86 \%(30 / 35,95 \%$ CI $71 \%$ to $94 \%$ ) in patients with normal-sized lymph nodes on CT, and $70 \%(31 / 45,95 \%$ CI $56 \%$ to $82 \%)$ in patients with enlarged lymph nodes. The prevalence of $\mathrm{N} 2-3$ disease was $11 \%(95 \% \mathrm{CI}$ $4 \%$ to $25 \%$ ) and $47 \%$ (95\% CI $33 \%$ to $61 \%$ ) in the two groups. Twenty-nine patients had normal-sized lymph nodes on CT as well as negative mediastinum on PET-CT (table 6); among these patients one had $\mathrm{N} 2-3$ as the final stage (false-negative rate $4 \%$ (95\% CI $0.01 \%$ to 0.18$)$ ).

Table 4 Tumour localisation at CT and N stage at PET-CT

\begin{tabular}{|c|c|c|c|c|}
\hline \multirow[b]{2}{*}{ PET-CT } & \multicolumn{4}{|l|}{$\mathrm{N}$-final } \\
\hline & $\overline{\text { NO-1 }}$ & N2-3 & $\mathrm{Nx}$ & Total \\
\hline \multicolumn{5}{|c|}{ Central tumour* } \\
\hline No-1 & 10 & 3 & 1 & 14 \\
\hline N2-3 & 3 & 5 & 2 & 10 \\
\hline \multicolumn{5}{|c|}{ Peripheral-intermediate $\dagger$} \\
\hline N0-1 & 33 & 5 & 1 & 39 \\
\hline N2-3 & 6 & 12 & 1 & 19 \\
\hline \multicolumn{5}{|c|}{ Not applicable, no information on exact tumour lacalisation } \\
\hline No-1 & - & - & - & \\
\hline N2-3 & 1 & 1 & - & 2 \\
\hline Total & & & & 84 \\
\hline
\end{tabular}

\section{Positive imaging and negative EUS-FNA}

Sixty-six patients had both CT and EUS-FNA performed, and 36 patients had both PET-CT and EUS-FNA performed. The yield of EUS-FNA and mediastinoscopy in the case of positive CT or PET-CT is illustrated in table 7. The NPV of EUS-FNA in the entire study population as well as in the subgroups with positive CT or PET-CT exceeds the NPV of mediastinoscopy. All patients with a positive PET-CT or CT and negative EUS-FNA but N2-3 as $\mathrm{N}$-final had a negative mediastinoscopy. Among the 24 patients who had N2-3 disease on CT, negative mediastinoscopy and $\mathrm{N} 2-3$ disease as $\mathrm{N}$-final, $\mathrm{N} 2-3$ was confirmed by EUS-FNA in 12 patients and by EBUS-TBNA in one. Similarly, EUS-FNA confirmed N2-3 disease in 7 of 10 patients with N2-3 disease on PET-CT, despite negative mediastinoscopy. Twenty-six patients had N2-3 disease on both CT and PET-CT; seven of these patients had final N0-1 (false-positive rate of $27 \%(95 \%$ CI $14 \%$ to $46 \%))$.

\section{DISCUSSION}

The results presented in this manuscript are based on an analysis of data on mediastinal staging from a prospective randomised clinical trial. The primary end point was futile thoracotomy and was previously reported. ${ }^{5}$ One of the strengths of our data is the

Table 5 Yield of invasive staging procedures in PET-CTnegative patients

\begin{tabular}{|c|c|c|c|c|}
\hline \multirow[b]{2}{*}{ PET-CT N0-1 $(n=53)$} & \multicolumn{4}{|l|}{$\mathrm{N}$-final } \\
\hline & No-1 & N2-3 & $\mathbf{N x}$ & Total \\
\hline \multicolumn{5}{|l|}{ Mediastinoscopy* } \\
\hline No-1 & 42 & 6 & 0 & 48 \\
\hline $\mathrm{N} 2-3$ & - & 1 & 0 & 1 \\
\hline \multirow[t]{2}{*}{$\begin{array}{l}\text { Not applicable, examination } \\
\text { not performed }\end{array}$} & 1 & 1 & 2 & 4 \\
\hline & & & & 53 \\
\hline \multicolumn{5}{|l|}{ EUS-FNA† } \\
\hline No-1 & 15 & 1 & 0 & 16 \\
\hline $\mathrm{N} 2-3$ & - & 4 & 0 & 4 \\
\hline \multirow[t]{2}{*}{$\begin{array}{l}\text { Not applicable, examination } \\
\text { not performed }\end{array}$} & 28 & 3 & 2 & 33 \\
\hline & & & & 53 \\
\hline
\end{tabular}


Table 6 Lymph node size and N stage at PET-CT

\begin{tabular}{rcccc}
\hline & N-final & & & \\
\cline { 2 - 5 } PET-CT & N0-1 & N2-3 & Nx & Total \\
\hline$<10 \mathrm{~mm}$ & & & & \\
N0-1 & 27 & 1 & 1 & 29 \\
N2-3 & 4 & 3 & 1 & 8 \\
& & & & 37 \\
$\geq 10 \mathrm{~mm}$ & & 7 & 1 & 24 \\
N0-1 & 16 & 15 & 2 & 23 \\
N2-3 & 6 & & & 47 \\
& & & & \\
\hline
\end{tabular}

consecutive and prospective recruitment of patients as well as the systematic application of mediastinoscopy, and to a certain extent, EUS-FNA, regardless of the results of the imaging test. However, the invasive diagnostic procedures have evolved significantly since the initiation of this study, especially with regard to a broader use of endoscopic techniques. The major limitation of this material is the relatively low number of patients, which particularly in subgroup analysis results in broad CIs and hence makes clear-cut conclusions difficult to achieve. Based on the presented results we will discuss some of the remaining problems in mediastinal staging of patients with lung cancer as outlined in the Introduction section.

\section{Diagnostic accuracy}

The overall diagnostic accuracy of a multimodality staging approach was improved by adding PET-CT. This is mainly based on the improved sensitivity of the PET-CT approach. Based on

Table 7 Results of invasive tests in CT or PET-CT negative patients

\begin{tabular}{|c|c|c|c|c|}
\hline & \multicolumn{4}{|l|}{$\mathrm{N}$-final } \\
\hline & $\overline{\mathrm{NO}-1}$ & N2-3 & Nx & Total \\
\hline \multicolumn{5}{|c|}{ (a) Yield of mediastinoscopy } \\
\hline \multicolumn{5}{|c|}{ CT N2-3 } \\
\hline \multicolumn{5}{|c|}{ Mediastinoscopy* } \\
\hline No-1† & 51 & 24 & 3 & 78 \\
\hline \multirow[t]{2}{*}{ N2-3 } & - & 15 & 0 & 15 \\
\hline & & & & 93 \\
\hline \multicolumn{5}{|c|}{ PET-CT N2-3 } \\
\hline \multicolumn{5}{|c|}{ Mediastinoscopy } \\
\hline No-1‡ & 9 & 10 & 2 & 21 \\
\hline \multirow[t]{2}{*}{ N2-3 } & - & 6 & 0 & 6 \\
\hline & & & & 27 \\
\hline \multicolumn{5}{|c|}{ (b) Yield of EUS-FNA } \\
\hline \multicolumn{5}{|c|}{ CT N2-3 } \\
\hline \multicolumn{5}{|l|}{ EUS-FNA§ } \\
\hline $\mathrm{N} 0-19$ & 20 & 5 & 0 & 25 \\
\hline \multirow[t]{2}{*}{$\mathrm{N} 2-3$} & - & 21 & 0 & 21 \\
\hline & & & & 46 \\
\hline \multicolumn{5}{|c|}{ PET-CT N2-3 } \\
\hline \multicolumn{5}{|c|}{ EUS-FNA } \\
\hline $\mathrm{N} 0-1^{* *}$ & 6 & 0 & 0 & 6 \\
\hline \multirow[t]{2}{*}{$\mathrm{N} 2-3$} & - & 10 & 0 & 10 \\
\hline & & & & 16 \\
\hline \multicolumn{5}{|c|}{ 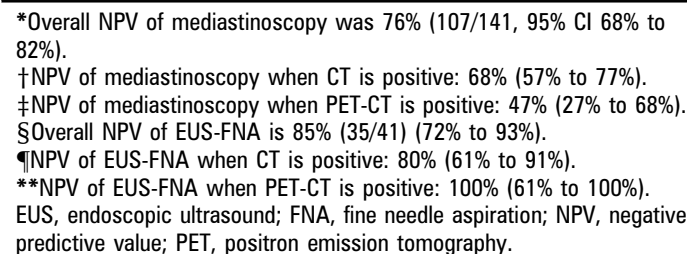 } \\
\hline
\end{tabular}

these findings as well as previously published studies demonstrating the ability of PET-CT to decrease the number of futile thoracotomies, ${ }^{5}{ }^{6}$ we suggest that PET-CT should be performed in all patients with NSCLC under consideration for surgery. However, in patients with centrally located primary tumours, PET-CT is only moderately better than chance in predicting the $\mathrm{N}$ stage $(\kappa=0.39)$.

\section{PET-negative lymph nodes}

Can mediastinoscopy be omitted in cases where there is no sign of mediastinal metastases on PET-CT? We found a frequency of false-negative mediastinum on PET-CT of $15 \%$ (8-27\%), roughly equal to what has been reported previously. ${ }^{7}$ In the case of a PET-CT-negative mediastinum, our data suggest EUS-FNA (false-negative rate of $6 \%$ ) as the most rational choice.

Adding a mediastinoscopy to a negative PET-CT hardly improves accuracy, as the false-negative rate in this setting was $13 \%$. It should however be noted that the sensitivity of mediastinoscopy in our material $(30 \%)$ is significantly below what has been previously reported in the literature. ${ }^{314}$ Two conditions in the present study can explain some of the divergence between the performance of mediastinoscopy in our study and what is generally published in the literature. First, the present study included mediastinoscopy, but did not focus on this examination. Thus the mediastinoscopies was done in a setting reflecting everyday routine and were not always done by dedicated experts. Further, the largest participating centre had an on-going trial during the study period, evaluating the diagnostic value of EUS-FNA, often performing EUS-FNA and mediastinoscopy during the same general anaesthesia, which could have resulted in a less thorough mediastinoscopy, for example after a positive EUS-FNA.

The impact of incidental N2 disease on patient prognosis can be disputed. In this study, two of eight patients with falsenegative mediastinal PET-CT had incidental N2 disease (found intraoperatively; surgery was completed with lymph node resection); both patients were alive at follow-up after 3 and 4.3 years.

\section{Lymph node size}

Two things demand attention when discussing the relationship of size of mediastinal lymph nodes to PET-CT findings: first the problem of partial volume effect, which can cause false-negative PET-CT results in smaller foci $(<10 \mathrm{~mm}),{ }^{15}$ potentially leading to understaging. Secondly the higher prevalence of malignancy in larger lymph nodes, which can affect the estimated PPV and NPV. In the present study we found a higher diagnostic accuracy of PET-CT assessing small lymph nodes compared with enlarged lymph nodes ( $>10 \mathrm{~mm}$ ) (86\% vs $70 \%)$ despite a lower prevalence of malignancy among the smaller nodules. A closer look reveals that sensitivity and specificity are similar in the two groups, but in concordance with the low prevalence NPV is significantly higher in the group with small nodules $(96 \%$ vs $70 \%$ ) and PPV is lower (43\% vs $71 \%$ ). Thus, in patients with normal-sized mediastinal lymph nodes a negative PET-CT is highly valid, whereas the risk of a false-negative diagnosis is substantial in enlarged lymph nodes without FDG uptake (30\%). It also demonstrates that the performance of PET-CT or any other test is never independent of the disease prevalence.

\section{Positive imaging and negative EUS-FNA}

Current recommendations suggest that in the case of positive findings on PET-CT or CT followed by a negative EUS-FNA or EBUS-TBNA, final confirmation by mediastinoscopy should be 
sought. ${ }^{2416}$ In the present study we did not find any additional value of mediastinoscopy in the case of positive imaging and negative EUS-FNA. However, as discussed above, the low sensitivity of mediastinoscopy in our study may not be representative for other settings. Further, the high accuracy of EUSFNA reported here might be difficult to achieve once the modality becomes part of daily clinical routine and is no longer performed solely by dedicated experts in the setting of an accuracy study. It should also be noted that the good performance of EUS-FNA in this study is based mainly on evaluation of lymph node station 7 (table 2) and not station $2-4 \mathrm{R}$ or $10-11$ which is better reached by EBUS. ${ }^{17}$

A meta-analysis has reported a relatively high false-negative rate of EUS-FNA of 19\%, compared with mediastinoscopy of $11 \%{ }^{4}$ However, the prevalence of N2-3 disease was higher in the EUS-FNA studies (61\%) compared with the mediastinoscopy studies (39\%). Two studies have looked at the diagnostic value of combined PET and EUS-FNA, finding an overall accuracy of $>90 \%$ and a false-negative rate of EUS-FNA in the case of a positive PET of $0-7 \%{ }^{18}$ This is confirmed by the present study where the false-negative rate of EUS-FNA after positive PET was zero, but $20 \%$ after a positive CT. Due to the relatively small sample size, this should be confirmed in a larger study.

\section{Conclusion}

In accordance with current recommendations we strongly recommend preoperative staging by PET-CT of patients with lung cancer. In patients without enlarged lymph nodes and a PET-negative mediastinum our data suggest that the patient may proceed directly to surgery. However, enlarged lymph nodes on CT needs confirmation independently of PET findings and a positive finding on PET-CT needs confirmation before a decision on surgery is made.

Funding Danish Cancer Society and the Danish Center for Health Technology Assessment.

Competing interests None.

Ethics approval This study was conducted with the approval of the Ethics Committee and Institutional Review Board of Copenhagen Hospitals.
Provenance and peer review Not commissioned; externally peer reviewed.

\section{REFERENCES}

1. Tanoue L. Staging of non-small cell lung cancer. Semin Respir Crit Care Med 2008:29:248-60

2. De Leyn P, Lardinois D, Van Schil P, et al. ESTS guidelines for preoperative lymph node staging for non-small cell lung cancer. Eur J Cardiothoracic Surg 2007;32:1-8.

3. Silvestri GA, Gould MK, Margolis ML, et al. Noninvasive staging of non-small cell lung cancer. ACCP evidenced-based clinical practice guidelines (2nd edition). Chest 2007;132(3 Suppl):178S-201S.

4. Detterbeck FC, Jantz MA, Wallace M, et al. Invasive mediastinal staging of lung cancer. ACCP evidence-based clinical practice guidelines (2nd edition). Chest 2007;132(3 Suppl):202s-20s.

5. Fischer B, Lassen U, Mortensen J, et al. Preoperative staging of lung cancer with combined PET-CT. N Engl J Med 2009;361:32-9.

6. Maziak DE, Darling GE, Inculet RI, et al. Positron emission tomography in staging early lung cancer. A randomized trial. Ann Intern Med 2009:151:221-8.

7. Al-Sarraf N, Aziz R, Gately K, et al. Pattern and predictors of occult mediastinal lymph node involvement in non-small cell lung cancer patients with negative mediastinal uptake on positron emission tomography. Eur J Cardiothoracic Surg 2008;33:104-9.

8. de Langen AJ, Raijmakers P, Riphagen I, et al. The size of mediastinal lymph nodes and its relation with metastatic involvement: a meta-analysis. Eur J Cardiothorac Surg 2006;29:26-9.

9. Danish Lung Cancer Group. Lung Cancer-Diagnosis and Therapy. Århus, Denmark: Dansk Lungecancer Gruppe, 2001. (ISBN: 87-988368-0-3)

10. Mountain CF. Revisions in the international system for staging lung cancer. Ches 1997:111:1710-17

11. Larsen SS, Vilmann P, Krasnik M, et al. Endoscopic ultrasound guided biopsy performed routinely in lung cancer staging spares futile thoracotomies: preliminary results from a randomised clinical trial. Lung Cancer 2005;49:377-85.

12. Altman DG. Comparing groups - categorical data. In: Altman DG, ed. Practical Statistics for Medical Research. London, UK: Chapman \& Hall, 1991:229-76.

13. Altman DG. Some common problems in medical research. In: Altman DG, ed Practical Statistics for Medical Research. London, UK: Chapman \& Hall, 1991:396-439.

14. Toloza EM, Harpole L, Detterbeck F, et al. Invasive staging of non-small cell lung cancer. Chest 2003;123(1 Suppl):157S-66S.

15. Fischer BM, Mortensen J. The future in diagnosis and staging of lung cancer: positron emission tomography. Respiration 2006;73:267-76.

16. Tournoy KG, De Ryck F, Vanwalleghem LR, et al. Endoscopic ultrasound reduces surgical mediastinal staging in lung cancer. A randomized trial. Am J Respir Crit Care Med 2008;177:531-5.

17. Vilmann $\mathbf{P}$, Annema J, Clementsen P. Endosonography in bronchopulmonary disease. Best Pract Res Clin Gastroenterol 2009:23:711-28.

18. Annema JT, Hoekstra OS, Smit EF, et al. Towards a minimally invasive staging strategy in NSCLC: analysis of PET positive mediastinal lesions by EUS-FNA. Lung Cancer 2004;44:53-60. 


\section{APPENDIX 1: DIAGNOSTIC ACCURACY IN ASSIGNING N0-1 VS N2-3}

\begin{tabular}{|c|c|c|c|c|c|}
\hline \multicolumn{3}{|l|}{ PET-CT } & \multicolumn{3}{|l|}{ CWU } \\
\hline Sensitivity & & $95 \% \mathrm{CI}$ & Sensitivity & & $95 \% \mathrm{CI}$ \\
\hline Consensus $\left(\mathrm{n}=92^{*}\right)$ & 0.75 & 0.59 to 0.86 & Consensus $(n=89 \dagger)$ & 0.59 & 0.41 to 0.74 \\
\hline PET-CT ( $\mathrm{n}=79 \neq)$ & 0.69 & 0.50 to 0.84 & & & \\
\hline CT $(n=92)$ & 0.78 & 0.62 to 0.88 & CT $(n=89)$ & 0.72 & 0.54 to 0.85 \\
\hline Mediastinoscopy $(n=86)$ & 0.28 & 0.16 to 0.45 & Mediastinoscopy ( $n=86$ ) & 0.38 & 0.23 to 0.56 \\
\hline EUS-FNA (n=39) & 0.94 & 0.73 to 0.99 & EUS-FNA $(n=29)$ & 0.67 & 0.42 to 0.84 \\
\hline Specificity & & & Specificity & & \\
\hline Consensus & 1 & 0.94 to 1 & Consensus & 0.98 & 0.91 to 1 \\
\hline PET-CT & 0.81 & 0.69 to 0.89 & & & \\
\hline CT & 0.52 & 0.39 to 0.64 & CT & 0.53 & 0.41 to 0.65 \\
\hline Mediastinoscopy & 1 & 0.93 to 1 & Mediastinoscopy & 0.98 & 0.91 to 1 \\
\hline EUS-FNA & 1 & 0.85 to 1 & EUS-FNA & 1 & 0.77 to 1 \\
\hline Positive predictive value & & & Positive predictive value & & \\
\hline Consensus & 1 & 0.88 to 1 & Consensus & 0.94 & 0.74 to 0.99 \\
\hline PET-CT & 0.64 & 0.46 to 0.79 & & & \\
\hline CT & 0.51 & 0.38 to 0.64 & CT & 0.43 & 0.30 to 0.57 \\
\hline Mediastinoscopy & 1 & 0.70 to 1 & Mediastinoscopy & 0.92 & 0.65 to 0.99 \\
\hline EUS-FNA & 1 & 0.80 to 1 & EUS-FNA & 1 & 0.72 to 1 \\
\hline Negative predictive value & & & Negative predictive value & & \\
\hline Consensus & 0.86 & 0.76 to 0.93 & Consensus & 0.83 & 0.73 to 0.90 \\
\hline PET-CT & 0.84 & 0.72 to 0.92 & & & \\
\hline CT & 0.78 & 0.63 to 0.89 & CT & 0.80 & 0.65 to 0.90 \\
\hline Mediastinoscopy & 0.70 & 0.59 to 0.79 & Mediastinoscopy & 0.76 & 0.65 to 0.84 \\
\hline EUS-FNA & 0.96 & 0.79 to 0.99 & EUS-FNA & 0.72 & 0.49 to 0.88 \\
\hline Accuracy & & & Accuracy & & \\
\hline Consensus & 0.90 & 0.82 to 0.95 & Consensus & 0.85 & 0.77 to 0.91 \\
\hline PET-CT & 0.77 & 0.67 to 0.85 & & & \\
\hline CT & 0.62 & 0.52 to 0.71 & CT & 0.60 & 0.49 to 0.69 \\
\hline Mediastinoscopy & 0.73 & 0.63 to 0.81 & Mediastinoscopy & 0.78 & 0.68 to 0.85 \\
\hline EUS-FNA & 0.97 & 0.87 to 1 & EUS-FNA & 0.82 & 0.64 to 0.92 \\
\hline
\end{tabular}

${ }^{*}$ Only patients with a final $\mathrm{N}$-stage.

†Only patients with a final $\mathrm{N}$-stage.

¥0f the included 98 patients, 13 did not have a PET-CT scan, five patients did not have a final N-stage and one patient did not have a PET-CT scan nor had assigned a final N-stage.

\section{Thorax online}

Visit Thorax online and listen to the latest podcast, post comments and download any you might have missed. Keep informed and up to date by visiting thorax.bmj.com. 